\title{
The approximation of reachable sets of control systems with integral constraint on controls
}

\author{
K. G. GUSEINOV \\ Department of Mathematics \\ Anadolu University \\ 26470, Eskisehir, Turkey \\ e-mail: kguseynov@anadolu.edu.tr \\ O. OZER \\ Department of Mathematics \\ Anadolu University \\ 26470, Eskisehir, Turkey \\ e-mail: oozer@anadolu.edu.tr \\ E. AKYAR \\ Department of Mathematics \\ Anadolu University \\ 26470, Eskisehir, Turkey \\ e-mail: eakyar@anadolu.edu.tr \\ V. N. USHAKOV \\ Institute for Mathematics and Mechanics \\ Ural Division of the Russian Academy of Science \\ S.Kovalevskaya st. 16 \\ Ekaterinburg 620066, Russia \\ e-mail: ushak@imm.uran.ru
}

\begin{abstract}
In this paper an approximation method for the construction of reachable sets of control systems with integral constraints on the control is considered. It is assumed that the control system is non-linear with respect to the phase state vector and is linear with respect to the control vector. The admissible control functions are chosen from the ball centered at the origin with radius $\mu_{0}$ in $L_{p}, p>1$. The reachable set is replaced by the set which
\end{abstract}


consists of finite number of points. The estimated accuracy of the Hausdorff distance between the reachable set and the set which is approximately constructed is obtained.

2000 Mathematics Subject Classification: Primary 34A34; Secondary 93 B03. Key words: Control system, Reachable set, Integral constraint.

\section{Introduction}

Numerous publications have been devoted to the study of various properties of the reachable sets of the control systems with geometrical constraints on control (see, e.g. $[1,4,5,14,15,18]$ and references therein). In papers (see, $[3,8,11,17]$ and references therein) the numerical methods for construction and estimation of the reachable sets of the control systems with geometrical constraints on control are considered. Control systems with integral constraints on control have been investigated in $[2,6,7,9,10,12,13,16]$.

Consider a control system whose behavior is described by a differential equation

$$
\dot{x}(t)=f(t, x(t))+B(t, x(t)) u(t),
$$

where $x \in R^{n}$ is the $n$-dimensional phase state vector, $u$ is the $r$-dimensional control vector, $t \in\left[t_{0}, \theta\right]\left(t_{0}<\theta<\infty\right)$ is the time, $f(t, x)$ is an $n$-dimensional vector function and $B(t, x)$ is an $(n \times r)$-dimensional matrix function.

It is assumed that the realizations $u(t), t \in\left[t_{0}, \theta\right]$, of the control $u$ are restricted by the constraint

$$
\int_{t_{0}}^{\theta}\|u(t)\|^{p} d t \leq \mu_{0}^{p}, \mu_{0}>0,1<p<\infty,
$$

where $\|\cdot\|$ denotes the Euclidean norm. It is also assumed that the following conditions are satisfied.

A. The functions $f(t, x)$ and $B(t, x)$ are continuous on $(t, x)$ and for any bounded set $D \subset\left[t_{0}, \theta\right] \times R^{n}$ there exist Lipschitz constants $L_{i}=L_{i}(D) \in$ $(0, \infty)(i=1,2)$ such that

$$
\begin{aligned}
\left\|f\left(t, x^{*}\right)-f\left(t, x_{*}\right)\right\| & \leq L_{1}\left\|x^{*}-x_{*}\right\|, \\
\left\|B\left(t, x^{*}\right)-B\left(t, x_{*}\right)\right\| & \leq L_{2}\left\|x^{*}-x_{*}\right\|
\end{aligned}
$$

for any $\left(t, x^{*}\right) \in D,\left(t, x_{*}\right) \in D$.

B. There exist constants $\gamma_{i} \in(0, \infty)(i=1,2)$ such that

$$
\|f(t, x)\| \leq \gamma_{1}(1+\|x\|),\|B(t, x)\| \leq \gamma_{2}(1+\|x\|)
$$

This research was supported by the Anadolu University Research Foundation and by the Russian Foundation for Basic Research. 
for every $(t, x) \in\left[t_{0}, \theta\right] \times R^{n}$. Here, $\|B\|$ is the Euclidean norm of matrix $B$.

Every function $u(\cdot) \in L_{p}\left(\left[t_{0}, \theta\right] ; R^{n}\right),(1<p<\infty)$, satisfying the inequality (1.2) is said to be an admissible control. By the symbol $U$ we denote the set of all admissible control functions $u(\cdot)$.

Let $u_{*}(\cdot) \in U$. The absolutely continuous function $x_{*}(t):\left[t_{0}, \theta\right] \rightarrow R^{n}$ which satisfies the equation $\dot{x}_{*}(t)=f\left(t, x_{*}(t)\right)+B\left(t, x_{*}(t)\right) u_{*}(t)$ a.e. in $\left[t_{0}, \theta\right]$, is said to be a solution of the system (1.1) generated by the admissible control function $u_{*}(\cdot)$. By the symbol $X\left(t_{0}, X_{0}\right)$ we denote the set of solutions of the system (1.1) which are generated by all possible admissible control functions $u(\cdot) \in U$ and satisfy initial condition $x_{*}\left(t_{0}\right) \in X_{0}$. We assume that

$$
\begin{gathered}
X\left(t ; t_{0}, X_{0}\right)=\left\{x(t) \in R^{n}: x(\cdot) \in X\left(t_{0}, X_{0}\right)\right\}, \\
H\left(t_{0}, X_{0}\right)=\left\{(t, x(t)) \in\left[t_{0}, \theta\right] \times R^{n}: x(\cdot) \in X\left(t_{0}, X_{0}\right)\right\} .
\end{gathered}
$$

$X\left(t ; t_{0}, X_{0}\right)$ is called the reachable set of the system (1.1) with initial set $\left(t_{0}, X_{0}\right)$ and constraint (1.2) at the instant of time $t$. The set $H\left(t_{0}, X_{0}\right)$ is called the integral funnel of the system (1.1) with constraint (1.2) and initial set $\left(t_{0}, X_{0}\right)$. It is obvious that the integral funnel can also be written as

$$
H\left(t_{0}, X_{0}\right)=\left\{(t, x) \in\left[t_{0}, \theta\right] \times R^{n}: x \in X\left(t ; t_{0}, X_{0}\right)\right\} .
$$

We consider approximation of the reachable set $X\left(\theta ; t_{0}, X_{0}\right)$. To solve the problem, the set of control functions $U$ is replaced by the set of controls $U_{1}$ which possess some geometrical constraints along with integral ones (Section 2). In the second step the set of controls $U_{1}$ is narrowed down to the set of controls $U_{2}$ which consists of piecewise constant controls (Section 3). In the third step the set of controls is replaced by the set of controls $U_{3}$ whose norms lie in uniform mesh of the given interval (Section 4). In the fourth step the set of controls $U_{3}$ is narrowed down to the set of controls $U_{4}$ which have finite number of control functions (Section 5) and finally the solutions of the differential equation is replaced by the Euler's broken lines (Section 6). Note that each subsequent set of controls is more convenient for approximation of the reachable set $X\left(\theta ; t_{0}, X_{0}\right)$. In section 7 the estimated accuracy of the Hausdorff distance between the reachable set $X\left(\theta ; t_{0}, X_{0}\right)$ and the set which is constructed approximately and consists of finite number of points, are given.

From now on it will be assumed that $X_{0} \subset R^{n}$ is compact set. For the following arguments, we will find the bounded cylinder $D \subset\left[t_{0}, \theta\right] \times R^{n}$ in which the integral funnel $H\left(t_{0}, X_{0}\right)$ is contained. Let

$$
\begin{aligned}
a & =\max \left\{\|x\|: x \in X_{0}\right\}, \\
r_{0} & =a+\gamma_{1}\left(\theta-t_{0}\right)+\mu_{0} \gamma_{2}\left(\theta-t_{0}\right)^{\frac{p-1}{p}}, \\
r & =r_{0}\left[1+\left(\gamma_{1}\left(\theta-t_{0}\right)+\mu_{0} \gamma_{2}\left(\theta-t_{0}\right)^{\frac{p-1}{p}}\right) e^{\gamma_{1}\left(\theta-t_{0}\right)+\mu_{0} \gamma_{2}\left(\theta-t_{0}\right)^{\frac{p-1}{p}}}\right], \\
D & =\left\{(t, x) \in\left[t_{0}, \theta\right] \times R^{n}:\|x\| \leq r\right\} .
\end{aligned}
$$


Proposition 1.1 The inclusion $H\left(t_{0}, X_{0}\right) \subset D$ holds where the cylinder $D$ is defined by relation (1.5).

The proof of the proposition can be obtained from the condition B.

Everywhere in the following arguments, we shall have in mind the cylinder (1.5) as the set $D$.

Let $\sigma>0$. Since $X_{0} \subset R^{n}$ is a compact set then it has a finite $\sigma$-net. Let $X_{0}^{\sigma}$ be a finite $\sigma$-net of $X_{0}$ and

$$
c_{*}=L_{1}\left(\theta-t_{0}\right)+L_{2} \mu_{0}\left(\theta-t_{0}\right)^{\frac{p-1}{p}} .
$$

We denote by

$$
\alpha(E, F)=\max \left\{\sup _{x \in F} d(x, E), \sup _{y \in E} d(y, F)\right\}
$$

the Hausdorff distance between the sets $E, F \subset R^{n}$ where $d(x, E)=\inf _{y \in E}\|x-y\|$.

Let us formulate a proposition which characterizes the estimated accuracy between the sets $X\left(t ; t_{0}, X_{0}\right)$ and $X\left(t ; t_{0}, X_{0}^{\sigma}\right)$.

\section{Proposition 1.2}

$$
\alpha\left(X\left(t ; t_{0}, X_{0}\right), X\left(t ; t_{0}, X_{0}^{\sigma}\right)\right) \leq \sigma\left(1+c_{*} e^{c_{*}}\right)
$$

for all $t \in\left[t_{0}, \theta\right]$.

The proof of the proposition follows from the condition A. Note, it is not difficult to verify that the set $X\left(t ; t_{0}, X_{0}\right)$ is closed and it depends on $t, t_{0}, X_{0}$ and $\mu_{0}$ continuously.

\section{Mixed constraints}

Suppose $\lambda \in(0, \infty)$. By $U_{1}$ we denote the set of all admissible control functions $u(\cdot) \in U$, which satisfy the geometrical constraint

$$
\|u(t)\| \leq \lambda, \quad t \in\left[t_{0}, \theta\right] .
$$

The set of all solutions of the system (1.1), satisfying initial condition $x\left(t_{0}\right) \in$ $X_{0}^{\sigma}$ and generated by all possible $u(\cdot) \in U_{1}$, is denoted by $X_{1}\left(t_{0}, X_{0}^{\sigma}\right)$. We set

$$
X_{1}\left(t ; t_{0}, X_{0}^{\sigma}\right)=\left\{x(t) \in R^{n}: x(\cdot) \in X_{1}\left(t_{0}, X_{0}^{\sigma}\right)\right\} .
$$

With the following proposition we will give the estimated accuracy of the Hausdorff distance between the sets $X\left(t ; t_{0}, X_{0}^{\sigma}\right)$ and $X_{1}\left(t ; t_{0}, X_{0}^{\sigma}\right)$.

Let

$$
\begin{aligned}
K_{1} & =\max _{(t, x) \in D}\|B(t, x)\|, \\
B_{1} & =\left\{x \in R^{n}:\|x\| \leq 1\right\} .
\end{aligned}
$$




\section{Proposition 2.1}

$$
\alpha\left(X\left(t ; t_{0}, X_{0}^{\sigma}\right), X_{1}\left(t ; t_{0}, X_{0}^{\sigma}\right)\right) \leq K_{1} \frac{2 \mu_{0}^{p}}{\lambda^{p-1}}\left(1+c_{*} e^{c_{*}}\right)
$$

for all $t \in\left[t_{0}, \theta\right]$ where $c_{*}$ is defined by (1.6).

Proof. Since $U_{1} \subset U$ then

$$
X_{1}\left(t ; t_{0}, X_{0}^{\sigma}\right) \subset X\left(t ; t_{0}, X_{0}^{\sigma}\right)
$$

for every $t \in\left[t_{0}, \theta\right]$. Choose an arbitrary $x(\cdot) \in X\left(t_{0}, X_{0}^{\sigma}\right)$. Then there exist $x_{0} \in X_{0}^{\sigma}$ and $u(\cdot) \in U$ such that

$$
x(t)=x_{0}+\int_{t_{0}}^{t} f(\tau, x(\tau))+B(\tau, x(\tau)) u(\tau) d \tau
$$

for all $t \in\left[t_{0}, \theta\right]$. Let us define the control function

$$
u_{*}(t)= \begin{cases}u(t) & \text { if }\|u(t)\| \leq \lambda, \\ \lambda \frac{u(t)}{\|u(t)\|} & \text { if }\|u(t)\|>\lambda\end{cases}
$$

where $t \in\left[t_{0}, \theta\right]$. It can be shown that $u_{*}(\cdot) \in U_{1}$. Let $x_{*}(\cdot):\left[t_{0}, \theta\right] \rightarrow R^{n}$ be the solution of the system (1.1) with initial condition $x_{*}\left(t_{0}\right)=x_{0}$, generated by control function $u_{*}(\cdot)$. Then $x_{*}(\cdot) \in X_{1}\left(t_{0}, X_{0}^{\sigma}\right)$ and taking into consideration the condition A, we obtain that

$$
\begin{aligned}
\left\|x(t)-x_{*}(t)\right\| \leq & \int_{t_{0}}^{t}\left(L_{1}+L_{2}\|u(\tau)\|\right)\left\|x(\tau)-x_{*}(\tau)\right\| d \tau \\
& +K_{1} \int_{t_{0}}^{t}\left\|u(\tau)-u_{*}(\tau)\right\| d \tau
\end{aligned}
$$

for every $t \in\left[t_{0}, \theta\right]$ where $K_{1}$ is defined by $(2.2)$.

Let us set $\Omega_{t}=\left\{\tau \in\left[t_{0}, t\right]:\|u(\tau)\|>\lambda\right\}$. Then, $\left[t_{0}, t\right] \backslash \Omega_{t}=\left\{\tau \in\left[t_{0}, t\right]:\right.$ $\|u(\tau)\| \leq \lambda\}$ and by definition of $u_{*}(\cdot),\left\|u(\tau)-u_{*}(\tau)\right\|=0$ when $\tau \in\left[t_{0}, t\right] \backslash \Omega_{t}$. We also have the inequality

$$
\begin{aligned}
\mu_{0}^{p} & \geq \int_{t_{0}}^{t}\|u(\tau)\|^{p} d \tau \geq \int_{\Omega_{t}}\|u(\tau)\|^{p} d \tau \\
& \geq \int_{\Omega_{t}} \lambda^{p} d \tau \geq \lambda^{p} \mu\left(\Omega_{t}\right),
\end{aligned}
$$

from which it follows that

$$
\mu\left(\Omega_{t}\right) \leq \frac{\mu_{0}^{p}}{\lambda^{p}}
$$


Here $\mu\left(\Omega_{t}\right)$ is the Lebesgue measure of the set $\Omega_{t}$. Using (2.5), Hölder's and Minkovski's inequalities we obtain

$$
\begin{aligned}
& \int_{t_{0}}^{t}\left\|u(\tau)-u_{*}(\tau)\right\| d \tau=\int_{\Omega_{t}}\left\|u(\tau)-u_{*}(\tau)\right\| d \tau \\
& \leq\left(\mu\left(\Omega_{t}\right)\right)^{\frac{p-1}{p}}\left[\left(\int_{\Omega_{t}}\|u(\tau)\|^{p} d \tau\right)^{\frac{1}{p}}+\left(\int_{\Omega_{t}}\left\|u_{*}(\tau)\right\|^{p} d \tau\right)^{\frac{1}{p}}\right] \\
& \quad \leq\left(\frac{\mu_{0}^{p}}{\lambda^{p}}\right)^{\frac{p-1}{p}}\left[\mu_{0}+\mu_{0}\right]=\frac{2 \mu_{0}^{p}}{\lambda^{p-1}} .
\end{aligned}
$$

Hence, by virtue of (2.4) and (2.6) we obtain

$$
\left\|x(t)-x_{*}(t)\right\| \leq K_{1} \frac{2 \mu_{0}^{p}}{\lambda^{p-1}}+\int_{t_{0}}^{t}\left(L_{1}+L_{2}\|u(\tau)\|\right)\left\|x(\tau)-x_{*}(\tau)\right\| d \tau .
$$

By the Gronwall's lemma it follows from here that

$$
\left\|x(t)-x_{*}(t)\right\| \leq K_{1} \frac{2 \mu_{0}^{p}}{\lambda^{p-1}}\left(1+c_{*} e^{c_{*}}\right), \quad t \in\left[t_{0}, \theta\right]
$$

where the constant $c_{*}$ is defined by the relation (1.6). Since $x(\cdot) \in X\left(t_{0}, X_{0}^{\sigma}\right)$ is an arbitrary solution, we have from (2.7) that

$$
X\left(t ; t_{0}, X_{0}^{\sigma}\right) \subset X_{1}\left(t ; t_{0}, X_{0}^{\sigma}\right)+K_{1} \frac{2 \mu_{0}^{p}}{\lambda^{p-1}}\left(1+c_{*} e^{c_{*}}\right) B_{1}, \quad t \in\left[t_{0}, \theta\right]
$$

The inclusions (2.3) and (2.8) complete the proof.

It follows from the Proposition 2.1 that

$$
\alpha\left(X\left(t ; t_{0}, X_{0}^{\sigma}\right), X_{1}\left(t ; t_{0}, X_{0}^{\sigma}\right)\right) \rightarrow 0 \text { as } \lambda \rightarrow \infty .
$$

\section{Piecewise constant controls}

Now we will define the new class of controls which consists of piecewise-constant controls on uniform partition of the interval $\left[t_{0}, \theta\right]$.

Let $\Gamma=\left\{t_{0}, t_{1}, \ldots, t_{N}=\theta\right\}$ be uniform partition of the interval $\left[t_{0}, \theta\right]$ such that

$$
t_{i+1}-t_{i}=\frac{\theta-t_{0}}{N}=\Delta, \quad i=0,1, \ldots, N-1
$$

Let

$$
U_{2}=\left\{u(\cdot) \in U_{1}: u(t)=u_{i} \forall t \in\left[t_{i}, t_{i+1}\right), i=0,1, \ldots, N-1\right\} .
$$


By the symbol $X_{2}\left(t_{0}, X_{0}^{\sigma}\right)$ we denote the set of solutions of the system (1.1) which are generated by all possible $u(\cdot) \in U_{2}$ and satisfy initial condition $x\left(t_{0}\right) \in$ $X_{0}^{\sigma}$. Now we set

$$
X_{2}\left(t ; t_{0}, X_{0}^{\sigma}\right)=\left\{x(t) \in R^{n}: x(\cdot) \in X_{2}\left(t_{0}, X_{0}^{\sigma}\right)\right\} .
$$

Assume that

$$
\begin{aligned}
K_{2} & =\max _{(t, x) \in D}\|f(t, x)\|, \\
\varphi(\Delta) & =K_{2} \Delta+K_{1} \mu_{0} \Delta^{\frac{p-1}{p}} .
\end{aligned}
$$

We formulate an auxiliary proposition which will be used in following arguments.

Proposition 3.1 The inequality

$$
\left\|x(t)-x\left(t_{i}\right)\right\| \leq \varphi(\Delta)
$$

holds for every $x(\cdot) \in X_{2}\left(t_{0}, X_{0}^{\sigma}\right), t \in\left[t_{i}, t_{i+1}\right]$ and $i=0,1, \ldots, N-1$.

Let us set

$$
\begin{gathered}
\omega^{*}(\Delta)=\max _{|t-\tau| \leq \Delta,\|x-y\| \leq \Delta}\|B(t, x)-B(\tau, y)\|, \quad(t, x) \in D,(\tau, y) \in D, \\
\xi(\Delta)=2 \mu_{0} \omega^{*}(\varphi(\Delta))\left(\theta-t_{0}\right)^{\frac{p-1}{p}}+2 \mu_{0} K_{1} \Delta^{\frac{p-1}{p}} .
\end{gathered}
$$

Now we give a proposition characterizing the Hausdorff distance between the sets $X_{1}\left(t ; t_{0}, X_{0}^{\sigma}\right)$ and $X_{2}\left(t ; t_{0}, X_{0}^{\sigma}\right)$.

Proposition 3.2 The inequality

$$
\alpha\left(X_{1}\left(t ; t_{0}, X_{0}^{\sigma}\right), X_{2}\left(t ; t_{0}, X_{0}^{\sigma}\right)\right) \leq \xi(\Delta)\left(1+c_{*} e^{c_{*}}\right)
$$

holds for all $t \in\left[t_{0}, \theta\right]$.

Here the constant $c_{*}$ is defined by relation (1.6).

Proof. Let us choose an arbitrary $x(\cdot) \in X_{1}\left(t_{0}, X_{0}^{\sigma}\right)$. Then, there exist $x_{0} \in X_{0}^{\sigma}$ and $u(\cdot) \in U_{1}$ such that

$$
x(t)=x_{0}+\int_{t_{0}}^{t} f(\tau, x(\tau))+B(\tau, x(\tau)) u(\tau) d \tau
$$

for all $t \in\left[t_{0}, \theta\right]$.

Now we define a new control $u_{*}(\cdot)$ using $u(\cdot)$ by the following equality

$$
u_{*}(t)=\frac{1}{\Delta} \int_{t_{i}}^{t_{i+1}} u(\tau) d \tau, \quad t \in\left[t_{i}, t_{i+1}\right) .
$$


It is not difficult to prove that $u_{*}(\cdot) \in U_{2}$. Let $x_{*}(\cdot)$ be the solution of the system (1.1) with initial condition $x\left(t_{0}\right)=x_{0}$, generated by the control $u_{*}(\cdot)$. Then $x_{*}(\cdot) \in X_{2}\left(t_{0}, X_{0}^{\sigma}\right)$ and taking into account the condition A we obtain

$$
\begin{aligned}
\left\|x(t)-x_{*}(t)\right\| \leq & \int_{t_{0}}^{t}\left(L_{1}+L_{2}\|u(\tau)\|\right)\left\|x(\tau)-x_{*}(\tau)\right\| d \tau \\
& +\left\|\int_{t_{0}}^{t} B\left(\tau, x_{*}(\tau)\right)\left(u(\tau)-u_{*}(\tau)\right) d \tau\right\|
\end{aligned}
$$

for all $t \in\left[t_{0}, \theta\right]$. Since $t \in\left[t_{0}, \theta\right]$, then there exists $k=0,1,2, \ldots, N-1$ such that $t \in\left[t_{k}, t_{k+1}\right)$ or $t=t_{N}=\theta$. Therefore,

$$
\begin{aligned}
& \int_{t_{0}}^{t} B\left(\tau, x_{*}(\tau)\right)\left(u(\tau)-u_{*}(\tau)\right) d \tau \\
& \quad=\sum_{i=0}^{k-1} \int_{t_{i}}^{t_{i+1}} B\left(\tau, x_{*}(\tau)\right)\left(u(\tau)-u_{*}(\tau)\right) d \tau \\
& \quad+\int_{t_{k}}^{t} B\left(\tau, x_{*}(\tau)\right)\left(u(\tau)-u_{*}(\tau)\right) d \tau
\end{aligned}
$$

holds. On the other hand, by the definition of $u_{*}(\cdot)$ we have

$$
\int_{t_{i}}^{t_{i+1}}\left(u(\tau)-u_{*}(\tau)\right) d \tau=0
$$

and consequently

$$
\begin{aligned}
& \int_{t_{i}}^{t_{i+1}} B\left(\tau, x_{*}(\tau)\right)\left(u(\tau)-u_{*}(\tau)\right) d \tau \\
& =\int_{t_{i}}^{t_{i+1}}\left[B\left(\tau, x_{*}(\tau)\right)-B\left(t_{i}, x_{*}\left(t_{i}\right)\right)\right]\left(u(\tau)-u_{*}(\tau)\right) d \tau
\end{aligned}
$$

is verified. Since $x_{*}(\cdot) \in X_{2}\left(t_{0}, X_{0}^{\sigma}\right)$, then according to Proposition 3.1 we have that

$$
\left\|x_{*}(t)-x_{*}\left(t_{i}\right)\right\| \leq \varphi(\Delta)
$$

for every $t \in\left[t_{i}, t_{i+1}\right)$ where $\varphi(\Delta)$ is defined by relation $(3.2)$ and $\varphi(\Delta)$ tends to zero as $\Delta \rightarrow 0$.

With no loss of generality we assume that $\Delta \leq \varphi(\Delta)$. Then it follows from (3.3) and (3.8) that

$$
\begin{aligned}
& \int_{t_{i}}^{t_{i+1}}\left\|B\left(\tau, x_{*}(\tau)\right)-B\left(t_{i}, x_{*}\left(t_{i}\right)\right)\right\|\left\|u(\tau)-u_{*}(\tau)\right\| d \tau \\
& \quad \leq \omega^{*}(\varphi(\Delta)) \int_{t_{i}}^{t_{i+1}}\left\|u(\tau)-u_{*}(\tau)\right\| d \tau
\end{aligned}
$$


holds. Consequently, applying Hölder's and Minkowski's inequalities we obtain that

$$
\begin{gathered}
\sum_{i=0}^{k-1} \int_{t_{i}}^{t_{i+1}}\left\|B\left(\tau, x_{*}(\tau)\right)\left(u(\tau)-u_{*}(\tau)\right)\right\| d \tau \\
\leq \sum_{i=0}^{k-1} \omega^{*}(\varphi(\Delta)) \int_{t_{i}}^{t_{i+1}}\left\|\left(u(\tau)-u_{*}(\tau)\right)\right\| d \tau \leq 2 \mu_{0} \omega^{*}(\varphi(\Delta))\left(t_{k}-t_{0}\right)^{\frac{p-1}{p}} \\
\int_{t_{k}}^{t}\left\|B\left(\tau, x_{*}(\tau)\right)\left(u(\tau)-u_{*}(\tau)\right)\right\| d \tau \leq 2 \mu_{0} K_{1} \Delta^{\frac{p-1}{p}}
\end{gathered}
$$

From (3.6), (3.7), (3.9) and (3.10) we conclude that

$$
\left\|\int_{t_{0}}^{t} B\left(\tau, x_{*}(\tau)\right)\left(u(\tau)-u_{*}(\tau)\right) d \tau\right\| \leq \xi(\Delta)
$$

where $\xi(\Delta)$ is defined by relation (3.4). Then, we have from (3.5) and (3.11) that

$$
\left\|x(t)-x_{*}(t)\right\| \leq \int_{t_{0}}^{t}\left(L_{1}+L_{2}\|u(\tau)\|\right)\left\|x(\tau)-x_{*}(\tau)\right\| d \tau+\xi(\Delta)
$$

holds for all $t \in\left[t_{0}, \theta\right]$. Finally, using Gronwall's lemma and the last inequality we get

$$
\left\|x(t)-x_{*}(t)\right\| \leq \xi(\Delta)\left(1+c_{*} e^{c_{*}}\right)
$$

for all $t \in\left[t_{0}, \theta\right]$ where $c_{*}>0$ is defined by (1.6). Since $x(\cdot) \in X_{1}\left(t_{0}, X_{0}^{\sigma}\right)$ is an arbitrary solution, it follows from (3.12) that

$$
X_{1}\left(t ; t_{0}, X_{0}^{\sigma}\right) \subset X_{2}\left(t ; t_{0}, X_{0}^{\sigma}\right)+\xi(\Delta)\left(1+c_{*} e^{c_{*}}\right) B_{1} .
$$

Since $U_{2} \subset U_{1}$, then (3.13) completes the proof.

We have from the Proposition 3.2 that

$$
\alpha\left(X_{1}\left(t ; t_{0}, X_{0}^{\sigma}\right), X_{2}\left(t ; t_{0}, X_{0}^{\sigma}\right)\right) \rightarrow 0 \text { as } \Delta \rightarrow 0 .
$$

\section{Piecewise constant controls with norms from uniform partition}

Let $\Gamma^{*}=\left\{y_{0}=0, y_{1}, \ldots, y_{R}=\lambda\right\}$ be uniform partition of the interval $[0, \lambda]$ such that

$$
y_{j+1}-y_{j}=\frac{\lambda}{R}=\Delta^{*} ; j=0,1,2, \ldots, R-1 .
$$


Let

$U_{3}=\left\{u(\cdot) \in U_{2}:\|u(t)\|=y_{j_{i}} \in \Gamma^{*} \forall t \in\left[t_{i}, t_{i+1}\right), i=0,1, \ldots, N-1\right\}$.

So, we narrow down the set of control functions $U_{2}$ to $U_{3}$ which consists of piecewise constant controls and the norms of which lie in a defined uniform partition $\Gamma^{*}$.

By the symbol $X_{3}\left(t_{0}, X_{0}^{\sigma}\right)$ we denote the set of all solutions of the system (1.1), which are generated by all possible $u(\cdot) \in U_{3}$ and satisfy initial condition $x\left(t_{0}\right) \in X_{0}^{\sigma}$. We set

$$
X_{3}\left(t ; t_{0}, X_{0}^{\sigma}\right)=\left\{x(t) \in R^{n}: x(\cdot) \in X_{3}\left(t_{0}, X_{0}^{\sigma}\right)\right\} .
$$

Let $u(\cdot) \in U_{3}$. Then, $\|u(t)\|=y_{j_{i}}$ for every $t \in\left[t_{i}, t_{i+1}\right)$ where $y_{j_{i}} \in \Gamma^{*}$ for any $i=0,1, \ldots, N-1$. Since the control function $u(\cdot) \in U_{3}$ satisfy the constraint (1.2) then we obtain the following inequality

$$
\sum_{i=0}^{N-1} \int_{t_{i}}^{t_{i+1}}\|u(\tau)\|^{p} d \tau=\Delta \sum_{i=0}^{N-1} y_{j_{i}}^{p} \leq \mu_{0}^{p}
$$

hence,

$$
\sum_{i=0}^{N-1} y_{j_{i}}^{p} \leq \frac{\mu_{0}^{p}}{\Delta}
$$

Therefore, for control functions $u(\cdot) \in U_{3}$ we have to use the constraint (4.1) instead of constraint (1.2).

Proposition 4.1 The inequality

$$
\alpha\left(X_{2}\left(t ; t_{0}, X_{0}^{\sigma}\right), X_{3}\left(t ; t_{0}, X_{0}^{\sigma}\right)\right) \leq K_{1} \Delta^{*}\left(\theta-t_{0}\right)\left(1+c_{*} e^{c_{*}}\right)
$$

holds for all $t \in\left[t_{0}, \theta\right]$.

The constants $K_{1}$ and $c_{*}$ are defined by relations (2.2) and (1.6) respectively.

Proof. Let $x(\cdot) \in X_{2}\left(t_{0}, X_{0}^{\sigma}\right)$ be an arbitrarily chosen solution. Then, there exist $x_{0} \in X_{0}^{\sigma}$ and $u(\cdot) \in U_{2}$ such that

$$
x(t)=x_{0}+\int_{t_{0}}^{t} f(\tau, x(\tau))+B(\tau, x(\tau)) u(\tau) d \tau
$$

for all $t \in\left[t_{0}, \theta\right]$.

Since $u(\cdot) \in U_{2}$ then, $u(t)=u_{i}$ for all $t \in\left[t_{i}, t_{i+1}\right)$ and $\left\|u_{i}\right\| \leq \lambda$ for each $i=0,1, \ldots, N-1$. Let us define the new control function $u_{*}(\cdot)$, setting

$$
u_{*}(t)= \begin{cases}y_{j_{i}} \frac{u_{i}}{\left\|u_{i}\right\|} & \text { if } 0<\left\|u_{i}\right\|<\lambda, \\ u_{i} & \text { if }\left\|u_{i}\right\|=0 \text { or }\left\|u_{i}\right\|=\lambda\end{cases}
$$


where $t \in\left[t_{i}, t_{i+1}\right), i=0,1, \ldots N-1$. Here $y_{j_{i}} \in \Gamma^{*}$ is such that $\left\|u_{i}\right\| \in\left[y_{j_{i}}, y_{j_{i+1}}\right)$. One can verify that $u_{*}(\cdot) \in U_{3}$ and the inequality

$$
\left\|u(t)-u_{*}(t)\right\| \leq \Delta^{*}
$$

holds for every $t \in\left[t_{0}, \theta\right]$. Suppose $x_{*}(\cdot)$ is the solution of the system (1.1) generated by control function $u_{*}(\cdot)$ which satisfies initial condition $x_{*}\left(t_{0}\right)=x_{0}$. Then, $x_{*}(\cdot) \in X_{3}\left(t_{0}, X_{0}^{\sigma}\right)$ and by virtue of condition A and (4.2) we obtain

$$
\left\|x(t)-x_{*}(t)\right\| \leq K_{1} \Delta^{*}\left(\theta-t_{0}\right)+\int_{t_{0}}^{t}\left(L_{1}+L_{2}\|u(\tau)\|\right)\left\|x(\tau)-x_{*}(\tau)\right\| d \tau
$$

for all $t \in\left[t_{0}, \theta\right]$ where $K_{1}$ is defined by (2.2). Now using Gronwall's lemma and Hölder's inequality we get

$$
\left\|x(t)-x_{*}(t)\right\| \leq K_{1} \Delta^{*}\left(\theta-t_{0}\right)\left(1+c_{*} e^{c_{*}}\right)
$$

for all $t \in\left[t_{0}, \theta\right]$ where $c_{*}$ is defined by relation (1.6). It means that the inclusion

$$
X_{2}\left(t ; t_{0}, X_{0}^{\sigma}\right) \subset X_{3}\left(t ; t_{0}, X_{0}^{\sigma}\right)+\left[K_{1} \Delta^{*}\left(\theta-t_{0}\right)\left(1+c_{*} e^{c_{*}}\right)\right] B_{1}
$$

holds for every $t \in\left[t_{0}, \theta\right]$. Since $U_{3} \subset U_{2}$, then (4.3) completes the proof of proposition.

It follows from the Proposition 4.1 that

$$
\alpha\left(X_{2}\left(t ; t_{0}, X_{0}^{\sigma}\right), X_{3}\left(t ; t_{0}, X_{0}^{\sigma}\right)\right) \rightarrow 0 \text { as } \Delta^{*} \rightarrow 0 .
$$

\section{$5 \quad$ Finite number of control functions}

Let $S=\left\{u \in R^{r}:\|u\|=1\right\}$ be the unit sphere of the space $R^{r}, \delta>0$ and $\widetilde{\Gamma}=\left\{s_{0}, s_{1}, \ldots, s_{p}\right\}$ be a finite $\delta$-net of $S$.

We now define the set of control functions $U_{4}$, which consists of finite number of control functions, setting

$$
\begin{aligned}
& U_{4}=\left\{u(\cdot) \in U_{3}:\right. \\
& \left.\quad u(t)=y_{j_{i}} s_{l_{i}}, \quad t \in\left[t_{i}, t_{i+1}\right), y_{j_{i}} \in \Gamma^{*}, s_{l_{i}} \in \widetilde{\Gamma}, i=0, \ldots, N-1\right\} .
\end{aligned}
$$

We denote by symbol $X_{4}\left(t_{0}, X_{0}^{\sigma}\right)$ the set of all solutions of the system (1.1), which are generated by the class of controls $U_{4}$ and satisfy initial condition $x\left(t_{0}\right) \in$ $X_{0}^{\sigma}$. Let

$$
X_{4}\left(t ; t_{0}, X_{0}^{\sigma}\right)=\left\{x(t) \in R^{n}: x(\cdot) \in X_{4}\left(t_{0}, X_{0}^{\sigma}\right)\right\},
$$

where $t \in\left[t_{0}, \theta\right]$. Since $X_{0}^{\sigma}$ is a finite $\sigma$-net of $X_{0}$, the set of control functions $U_{4}$ consists of finite number of control functions, therefore the set $X_{4}\left(t ; t_{0}, X_{0}^{\sigma}\right)$ includes finite number of points. 
Proposition 5.1 The following inequality holds

$$
\alpha\left(X_{3}\left(t ; t_{0}, X_{0}^{\sigma}\right), X_{4}\left(t ; t_{0}, X_{0}^{\sigma}\right)\right) \leq K_{1} \delta \lambda\left(\theta-t_{0}\right)\left(1+c_{*} e^{c_{*}}\right)
$$

for all $t \in\left[t_{0}, \theta\right]$.

The positive constants $K_{1}$ and $c_{*}$ are defined by relations (1.6) and (2.2) respectively.

Proof. Let us choose an arbitrary $x(\cdot) \in X_{3}\left(t_{0}, X_{0}^{\sigma}\right)$. Then, there exist $x_{0} \in X_{0}^{\sigma}$ and $u(\cdot) \in U_{3}$ such that

$$
x(t)=x_{0}+\int_{t_{0}}^{t} f(\tau, x(\tau))+B(\tau, x(\tau)) u(\tau) d \tau
$$

for all $t \in\left[t_{0}, \theta\right]$

Since $u(\cdot) \in U_{3}$ then, by the definition of $U_{3}, u(\cdot)$ can be written as

$$
u(t)=y_{j_{i}} b_{l_{i}}, \quad y_{j_{i}} \in \Gamma^{*}, \quad b_{l_{i}} \in S
$$

for all $t \in\left[t_{i}, t_{i+1}\right)(i=0,1, \ldots, N-1)$.

By virtue of the definition of the $\delta$-net, for each $b_{l_{i}} \in S$ there exists $s_{l_{i}} \in \widetilde{\Gamma}$ such that $\left\|b_{l_{i}}-s_{l_{i}}\right\| \leq \delta$. Let

$$
u_{*}(t)=y_{j_{i}} s_{l_{i}}, \quad y_{j_{i}} \in \Gamma^{*}, \quad s_{l_{i}} \in \widetilde{\Gamma}, \quad t \in\left[t_{i}, t_{i+1}\right), \quad i=0,1, \ldots, N-1 .
$$

It is obvious that $u_{*}(\cdot) \in U_{4}$ and the inequality

$$
\left\|u(t)-u_{*}(t)\right\| \leq \delta \lambda
$$

holds for all $t \in\left[t_{0}, \theta\right]$.

Suppose $x_{*}(\cdot)$ is the solution of the system (1.1) generated by the control function $u_{*}(\cdot) \in U_{4}$, which satisfies initial condition $x\left(t_{0}\right)=x_{0}$. Then, by virtue of condition A and inequality (5.1) we have that

$$
\left\|x(t)-x_{*}(t)\right\| \leq \int_{t_{0}}^{t}\left(L_{1}+L_{2}\|u(\tau)\|\right)\left\|x(\tau)-x_{*}(\tau)\right\| d \tau+\int_{t_{0}}^{t} K_{1} \delta \lambda d \tau
$$

for all $t \in\left[t_{0}, \theta\right]$. Then, applying Gronwall's lemma we obtain

$$
\left\|x(t)-x_{*}(t)\right\| \leq K_{1} \delta \lambda\left(\theta-t_{0}\right)\left(1+c_{*} e^{c_{*}}\right), \quad t \in\left[t_{0}, \theta\right] .
$$

Thus,

$$
X_{3}\left(t ; t_{0}, X_{0}^{\sigma}\right) \subset X_{4}\left(t ; t_{0}, X_{0}^{\sigma}\right)+\left[K_{1} \delta \lambda\left(\theta-t_{0}\right)\left(1+c_{*} e^{c_{*}}\right)\right] B_{1}
$$

for every $t \in\left[t_{0}, \theta\right]$. Since $U_{4} \subset U_{3}$, from (5.2) we complete the proof.

We obtain from Proposition 5.1 that for every $\varepsilon>0$ and $\lambda>0$ there exist $\delta>0$ such that

$$
\alpha\left(X_{3}\left(t ; t_{0}, X_{0}^{\sigma}\right), X_{4}\left(t ; t_{0}, X_{0}^{\sigma}\right)\right) \leq \varepsilon .
$$




\section{Euler's method}

Now we will approximately calculate the set $X_{4}\left(\theta ; t_{0}, X_{0}^{\sigma}\right)$. Same arguments and estimates are valid for the general case $X_{4}\left(t ; t_{0}, X_{0}^{\sigma}\right), t \in\left[t_{0}, \theta\right]$.

We will construct the Euler's broken line

$$
\begin{gathered}
z(t)=z\left(t_{i}\right)+\left(t-t_{i}\right)\left[f\left(t_{i}, z\left(t_{i}\right)\right)+B\left(t_{i}, z\left(t_{i}\right)\right) u\left(t_{i}\right)\right], \\
z\left(t_{0}\right)=x\left(t_{0}\right)=x_{0} \in X_{0}^{\sigma}, \quad t \in\left[t_{i}, t_{i+1}\right), \quad i=0,1, \ldots, N-1,
\end{gathered}
$$

which corresponds to solution $x(\cdot)$ of the system (1.1), generated by a control function $u(\cdot) \in U_{4}$, where $x\left(t_{0}\right)=x_{0}$. We will denote by the symbol $Z\left(\theta ; t_{0}, X_{0}^{\sigma}\right)$ the set of values of $z(\theta)$ of the Euler's broken lines, generated by all possible controls of $U_{4}$ from all initial positions $x_{0} \in X_{0}^{\sigma}$. formula

The values of $z(\theta) \in Z\left(\theta ; t_{0}, X_{0}^{\sigma}\right)$ can be calculated by the following recursive

$$
\begin{array}{r}
z\left(t_{i+1}\right)=z\left(t_{i}\right)+\Delta\left[f\left(t_{i}, z\left(t_{i}\right)\right)+B\left(t_{i}, z\left(t_{i}\right)\right) y_{j_{i}} s_{l_{i}}\right], \\
z\left(t_{0}\right)=x_{0} \in X_{0}^{\sigma}, \quad y_{j_{i}} \in \Gamma^{*}, \quad s_{l_{i}} \in \widetilde{\Gamma}, \quad i=0,1, \ldots, N-1,
\end{array}
$$

where the numbers $y_{j_{i}} \in \Gamma^{*}$ satisfy the inequality (4.1). Assume

$$
\begin{gathered}
K^{*}(\Delta)=\max _{|\tau-t| \leq \Delta,\|y-x\| \leq \Delta}\|f(\tau, y)-f(t, x)\|, \quad(\tau, y) \in D, \quad(t, x) \in D \\
\eta^{*}(\lambda, \Delta)=K^{*}(\varphi(\Delta))+\lambda \omega^{*}(\varphi(\Delta)) \\
\eta(\lambda, \Delta)=\Delta \eta^{*}(\lambda, \Delta), \quad L(\lambda)=L_{1}+L_{2} \lambda \\
g(\lambda)=\left(\theta-t_{0}\right) e^{L(\lambda)\left(\theta-t_{0}\right)}
\end{gathered}
$$

The functions $\varphi(\Delta)$ and $\omega^{*}(\Delta)$ are defined by relations (3.2) and (3.3) respectively.

Proposition 6.1 The inequality

$$
\alpha\left(X_{4}\left(\theta ; t_{0}, X_{0}^{\sigma}\right), Z\left(\theta ; t_{0}, X_{0}^{\sigma}\right)\right) \leq g(\lambda) \eta^{*}(\lambda, \Delta)
$$

holds.

Proof. Let $x(\cdot)$ be an arbitrary solution of the system (1.1), generated by the control function $u(\cdot) \in U_{4}$ which satisfies initial condition $x\left(t_{0}\right)=x_{0} \in X_{0}^{\sigma}$ where $u(t)=y_{j_{i}} s_{l_{i}}$ as $t \in\left[t_{i}, t_{i+1}\right), y_{j_{i}} \in \Gamma^{*}, s_{l_{i}} \in \widetilde{\Gamma}, i=0,1, \ldots, N-1$. 
Suppose $\varepsilon_{1}=\left\|x\left(t_{1}\right)-z\left(t_{1}\right)\right\|$. Then according to the Proposition 6.1 and (6.1), the inequality

$$
\begin{aligned}
\varepsilon_{1} \leq & \int_{t_{0}}^{t_{1}}\left\|f(\tau, x(\tau))-f\left(t_{0}, x\left(t_{0}\right)\right)\right\| d \tau \\
& +\int_{t_{0}}^{t_{1}}\left\|B(\tau, x(\tau))-B\left(t_{0}, x\left(t_{0}\right)\right)\right\|\left\|y_{j_{0}} s_{l_{0}}\right\| d \tau \\
\leq & \int_{t_{0}}^{t_{1}} K^{*}(\varphi(\Delta)) d \tau+\int_{t_{0}}^{t_{1}} \lambda \omega^{*}(\varphi(\Delta)) d \tau=\Delta \eta^{*}(\lambda, \Delta)=\eta(\lambda, \Delta)
\end{aligned}
$$

holds.

Now, let $\varepsilon_{2}=\left\|x\left(t_{2}\right)-z\left(t_{2}\right)\right\|$. Then it follows from (6.1), (6.4) and Proposition 3.1 that

$$
\begin{gathered}
\left\|x\left(t_{2}\right)-z\left(t_{2}\right)\right\| \leq\left\|x\left(t_{1}\right)-z\left(t_{1}\right)\right\|+\| \int_{t_{1}}^{t_{2}}\left[f(\tau, x(\tau))+B(\tau, x(\tau)) y_{j_{1}} s_{\ell_{1}}\right] d \tau \\
-\Delta\left[f\left(t_{1}, z\left(t_{1}\right)\right)+B\left(t_{1}, z\left(t_{1}\right)\right) y_{j_{1}} s_{\ell_{1}}\right] \| \\
\leq \eta(\lambda, \Delta)+\int_{t_{1}}^{t_{2}}\left\|f(\tau, x(\tau))-f\left(t_{1}, z\left(t_{1}\right)\right)\right\| d \tau \\
\quad+\lambda \int_{t_{1}}^{t_{2}}\left\|B(\tau, x(\tau))-B\left(t_{1}, z\left(t_{1}\right)\right)\right\| d \tau \\
\leq \eta(\lambda, \Delta)+\int_{t_{1}}^{t_{2}}\left\|f(\tau, x(\tau))-f\left(t_{1}, x\left(t_{1}\right)\right)\right\| d \tau \\
\quad+\int_{t_{1}}^{t_{2}}\left\|f\left(t_{1}, x\left(t_{1}\right)\right)-f\left(t_{1}, z\left(t_{1}\right)\right)\right\| d \tau \\
+\lambda\left(\int_{t_{1}}^{t_{2}}\left\|B(\tau, x(\tau))-B\left(t_{1}, x\left(t_{1}\right)\right)\right\| d \tau+\int_{t_{1}}^{t_{2}}\left\|B\left(t_{1}, x\left(t_{1}\right)\right)-B\left(t_{1}, z\left(t_{1}\right)\right)\right\| d \tau\right) \\
\leq \eta(\lambda, \Delta)+\int_{t_{1}}^{t_{2}} K^{*}(\varphi(\Delta)) d \tau+L_{1} \int_{t_{1}}^{t_{2}}\left\|x\left(t_{1}\right)-z\left(t_{1}\right)\right\| d \tau \\
+\lambda\left(\int_{t_{1}}^{t_{2}} \omega^{*}(\varphi(\Delta)) d \tau+L_{2} \int_{t_{1}}^{t_{2}}\left\|x\left(t_{1}\right)-z\left(t_{1}\right)\right\| d \tau\right) \\
\leq \eta(\lambda, \Delta)+\Delta K^{*}(\varphi(\Delta))+L_{1} \Delta \eta(\lambda, \Delta)+\lambda\left[\Delta \omega^{*}(\varphi(\Delta))+L_{2} \Delta \eta(\lambda, \Delta)\right] .
\end{gathered}
$$

Since $\eta(\lambda, \Delta)=\Delta \eta^{*}(\lambda, \Delta)=\Delta\left[K^{*}(\varphi(\Delta))+\lambda \omega^{*}(\varphi(\Delta))\right], L(\lambda)=L_{1}+\lambda L_{2}$ and $1+L(\lambda) \Delta \leq \exp (L(\lambda) \Delta)$, we get

$$
\begin{aligned}
\left\|x\left(t_{2}\right)-z\left(t_{2}\right)\right\| & \leq \eta(\lambda, \Delta)+\eta(\lambda, \Delta)+L_{1} \Delta \eta(\lambda, \Delta)+\lambda L_{2} \Delta \eta(\lambda, \Delta) \\
& =\eta(\lambda, \Delta)+\eta(\lambda, \Delta)\left(1+L_{1} \Delta+\lambda L_{2} \Delta\right) \\
& =\eta(\lambda, \Delta)+\eta(\lambda, \Delta)(1+L(\lambda) \Delta) \\
& \leq \eta(\lambda, \Delta)+\eta(\lambda, \Delta) \exp (L(\lambda) \Delta) .
\end{aligned}
$$

Similarly, the estimation

$$
\varepsilon_{3} \leq \eta(\lambda, \Delta) e^{L(\lambda)(\Delta+\Delta)}+\eta(\lambda, \Delta) e^{L(\lambda) \Delta}+\eta(\lambda, \Delta)
$$

holds for $\varepsilon_{3}=\left\|x\left(t_{3}\right)-z\left(t_{3}\right)\right\|$. 
Finally, for $\varepsilon_{N}=\left\|x\left(t_{N}\right)-z\left(t_{N}\right)\right\|=\|x(\theta)-z(\theta)\|$, we obtain

$$
\varepsilon_{N} \leq e^{L(\lambda)\left(\theta-t_{0}\right)} \sum_{i=0}^{N-1} \eta(\lambda, \Delta)=g(\lambda) \eta^{*}(\lambda, \Delta) .
$$

This completes the proof.

It follows from Proposition 6.1 that for every $\varepsilon>0$ and $\lambda>0$ there exist $\Delta>0$ such that

$$
\alpha\left(X_{4}\left(\theta ; t_{0}, X_{0}^{\sigma}\right), Z\left(\theta ; t_{0}, X_{0}^{\sigma}\right)\right) \leq \varepsilon
$$

\section{Main result}

From Propositions 1.2, 2.1, 3.2, 4.1, 5.1 and 6.1 we obtain the validity of the following theorem which gives evaluated accuracy of Hausdorff distance between the reachable set $X\left(\theta ; t_{0}, X_{0}\right)$ of the system (1.1) generated by the control functions $u(\cdot) \in U$ and the set $Z\left(\theta ; t_{0}, X_{0}^{\sigma}\right)$ which consists of finite number of points.

Theorem 7.1 The estimation

$$
\begin{aligned}
\alpha\left(X\left(\theta ; t_{0}, X_{0}\right), Z\left(\theta ; t_{0}, X_{0}^{\sigma}\right)\right) \leq & \sigma\left(1+c_{*} e^{c_{*}}\right)+K_{1} \frac{2 \mu_{0}^{p}}{\lambda^{p-1}}\left(1+c_{*} e^{c_{*}}\right) \\
& +\xi(\Delta)\left(1+c_{*} e^{c_{*}}\right)+K_{1} \Delta^{*}\left(\theta-t_{0}\right)\left(1+c_{*} e^{c_{*}}\right) \\
& +K_{1} \delta \lambda\left(\theta-t_{0}\right)\left(1+c_{*} e^{c_{*}}\right)+g(\lambda) \eta^{*}(\lambda, \Delta)
\end{aligned}
$$

holds where $c_{*}, K_{1}, \xi(\Delta), \eta^{*}(\lambda, \Delta)$ and $g(\lambda)$ are defined by relations (1.6), (2.2), (3.4), (6.2) and (6.3) respectively.

Note that if the system $(1.1)$ is autonomous i.e., if the functions $(t, x) \rightarrow$ $f(t, x)$ and $(t, x) \rightarrow B(t, x)$ do not depend on $t$ then

$$
\begin{gathered}
\xi(\Delta)=2 \mu_{0} L_{2} \varphi(\Delta)\left(\theta-t_{0}\right)^{\frac{p-1}{p}}+2 \mu_{0} K_{1} \Delta^{\frac{p-1}{p}} \\
\eta^{*}(\lambda, \Delta)=\left(L_{1}+\lambda L_{2}\right) \varphi(\Delta)
\end{gathered}
$$

where $\varphi(\Delta)$ is defined by $(3.2)$.

The validity of the following corollary follows from Theorem 7.1.

Corollary 7.2 For any $\varepsilon>0$ there exist $\sigma>0, \lambda>0, \Delta>0, \Delta^{*}>0, \delta>0$ such that

$$
\alpha\left(X\left(\theta ; t_{0}, X_{0}\right), Z\left(\theta ; t_{0}, X_{0}^{\sigma}\right)\right) \leq \varepsilon
$$




\section{References}

[1] V. I. BLAGODATSKIKH and A. F. FILIPPOV, Differential inclusions and optimal control, Proc. of the Steklov Inst. of Math. 169 (1986), 199-256.

[2] A. G. CHENTSOV, Asymptotic attainability with perturbation of integral constraints, Cybernet. Sistems Anal., 31 (1995), 75-84.

[3] F. L. CHERNOUSKO, State Estimation for Dynamic Systems, CRC Press, Boca Raton, 1994.

[4] F. H. CLARKE A proximal characterization of the reachable set, Systems Control Lett., 27 (1996), 195-197.

[5] F. H. CLARKE, YU. S. LEDYAYEV, R. J. STERN and P. R. WOLENSKI, Nonsmooth Analysis and Control Theory, Springer-Verlag, New York, 1998.

[6] R. CONTI, "Problemi di controllo e di controllo ottimale", UTET, Torino, 1974.

[7] F. GOZZI, P. LORETI, Regularity of the minimum time function and minimum energy problems: the linear case, SIAM J. Control Optim. 37 (1999) $1195-1221$.

[8] KH. G. GUSEINOV, A. N. MOISEYEV, and V. N. USHAKOV, The approximation of reachable domains of control systems, J. Appl. Math. Mech., 62 (1998), 169-175.

[9] KH. G. GUSEINOV, O. OZER, and E. AKYAR, On the continuity properties of the attainable sets of control systems with integral constraints on control, Nonlinear Anal. Ser. A: Theory, Meth., Appl., 56 (2004), 433-449.

[10] M. R. JAMES, and I. R. PETERSEN, Nonlinear state estimation for uncertain systems with an integral constraints, IEEE Trans. Signal Process., 46 (1998), 2926-2937.

[11] A. B. KURZHANSKI, and I. VALYI, Ellipsoidal Calculus for Estimation and Control, Birkhauser, Boston, 1996.

[12] M. MOTTA, and F. RAMPAZZO, Multivalued dynamics on a closed domain with absorbing boundary, applications to optimal control problems with integral constraints, Nonlinear Anal. Ser. A: Theory, Meth., Appl., 41 (2000), 631-647.

[13] M. MOTTA, and C. SARTORI, Minimum time with bounded energy, minimum energy with bounded time, SIAM J. Control Optim., 42 (2003), 789-809. 
[14] A. I. PANASYUK, Equations of attainable set dynamics, part 1: Integral funnel equation, J. Optim. Theory Appl., 64 (1990), 349-366.

[15] T. RZEZUCHOWSKI, Continuous parametrization of attainable sets by solutions of differential inclusions, Set Valued Anal., 7 (1999), 347-355.

[16] P. SORAVIA, Viscosity solutions and optimal control problems with integral constraints, Systems Control Lett., 40 (2000), 325-335.

[17] V. N. USHAKOV, and A. P. KHRIPUNOV, The approximate construction of integral cones of differential inclusions, Zh. Vychisl. Mat. Mat. Fiz., 34 (1994), 965-977.

[18] P. WOLENSKI, The exponential formula for the reachable set of Lipschitz differential inclusion, SIAM J. Control Optim. 28 (1990), 1148-1161.

Received 13 May 2004; accepted 15 October 2004

Published Online First 13 August 2007

To access this journal online:

http://www.birkhauser.ch 